$\underline{\mathbf{P}-22}$

\title{
Theoretical and Experimantal Studies on the Performance of TBHQ and BHA as a Chain-Breaking Radical Scavenger
}

\author{
Siti Balqis Che Othman, Juriffah Ariffin and Ku Halim Ku Bulat ${ }^{*}$ \\ Department of Chemical Sciences, Faculty Science of Technology, University Malaysia Terengganu 21030, Kuala \\ Terengganu, Terengganu Darul Iman; E-mail: keriang@umt.edu.my
}

The aim of this research project is to study the performance of two antioxidants, TBHQ (1) and BHA (2) as a chain-breaking radical scavenger of the selected vegetable oil which contained high oleic fatty acid constituent i.e. canola oil. Experimental studies involved heating the vegetable oil in the forced air-flow oven for 15 days at $60^{\circ} \mathrm{C}$. Samples subjected for peroxide value determination were analysed at $0,1,3,6,10$ and 15 days of exposure. For the theoretical investigation, Gaussian 09 software package at the theoretical level of DFT B3LYP/6-31G(d,p) were used to evaluate the physical parameters of the antioxidants (TBHQ and BHA), the homo-chain of tri-oleic TAG (3) with hydroperoxide group at carbon-11 and its radical, and the transition-state complex of the two components in questions. The selected physical parameters such as the total electronic energy (SCF energy), the stabilization energy, the hydrogen bond distance of the complex, the properties of the O-H of antioxidant (bond length, bond dissociation energy, Wiberg bond-index), and the dipole moment, were then utilized to correlate with the actual performance of the two antioxidants. Experimental results showed that TBHQ exhibit better performance than BHA in inhibiting the production of hydroperoxides in canola oil. These experimental observations were in line with the theoretical results where all the physical parameters except hydrogen bond distance support the findings.

Keywords: Canola oil, TBHQ, BHA, Tri-oleic, theoretical studies. 